Barragán, R.; González-Ravé, J.M.; González-Mohíno, F.; Yustres, I.; Juárez Santos-García, D. (2020) Effects of Swimming Intensity on Triathlon Performance. Revista Internacional de Medicina y Ciencias de la Actividad Física y el Deporte vol. 20 (80) pp. 529-538 Http://cdeporte.rediris.es/revista/revista80/artefectos1208.htm

DOI: https://doi.org/10.15366/rimcafd2020.80.004

\title{
ORIGINAL
}

\section{EFECTOS DE LA INTENSIDAD DE NADO EN EL RENDIMIENTO DEL TRIATLON}

\section{EFFECTS OF SWIMMING INTENSITY ON TRIATHLON PERFORMANCE}

\author{
Barragán, R. ${ }^{1}$; González-Ravé, J.M. ${ }^{1}$; González-Mohíno, F. ${ }^{1,2}$; Yustres, I. ${ }^{1}$ y \\ Juárez Santos-García, D. ${ }^{1}$
${ }^{1}$ Laboratorio Entrenamiento Deportivo, Facultad Ciencias del Deporte, Universidad Castilla-La Mancha, Toledo (España) rubenbarragancastellanos@hotmail.com, josemaria.gonzalez@uclm.es, inmaculada.yustres@uclm.es, daniel.juarez@uclm.es.
${ }^{2}$ Facultad de Lenguas y Educación, Universidad Nebrija, Madrid (España) fgonzalezmohino@nebrija.es.

Código UNESCO/UNESCO code: 2411 Fisiología del Ejercicio / Exercise Physiology.

Clasificación Consejo de Europa / Council of Europe Classification: 6. Fisología del Ejercicio / Exercise Physiology.

Recibido 15 de enero de 2019 Received January 15, 2019 Aceptado 25 marzo de 2019 Accepted March 25, 2019

\section{RESUMEN}

Objetivo: Analizar la influencia de diferentes intensidades de nado en los sectores de ciclismo y carrera a pie y en el rendimiento final del triatlón sprint. Métodos: Siete triatletas sub23 y Absolutos (altura de 1,74 $\pm 0,04 \mathrm{~m}$, peso de $70,82 \pm 6,76 \mathrm{~kg}$, edad de $23,42 \pm 3,25$ años, $\mathrm{VO}_{2}$ max de $63,54 \pm 5,23 \mathrm{ml} \cdot \mathrm{kg}^{-}$ $\left.{ }^{1} \cdot \mathrm{min}^{-1}\right)$ participaron en este estudio. Realizaron tres triatlones completos a intensidades de nado diferentes (70\%, $80 \%$ y $90 \%$ de un test de $750 \mathrm{~m}$ previo). Se midió la frecuencia cardíaca y el lactato al finalizar cada sector y el triatlón completo. Resultados: La intensidad de nado del $90 \%$ obtuvo el mejor rendimiento final. El lactato y frecuencia cardíaca en el sector de nado para esta condición incrementó significativamente, sin diferencias en los sectores siguientes. Conclusiones: Basándonos en la muestra estudiada, el rendimiento final en un triatlón sprint parece estar condicionado por la intensidad de nado, siendo el $90 \%$ la mejor intensidad observada en triatletas moderadamente entrenados. 
PALABRAS CLAVE: resistencia, rendimiento, entrenamiento.

\begin{abstract}
Objective: To analyze the influence of different swimming intensities on the subsequent cycling and running sectors and overall sprint triathlon performance. Methods: Seven sub23 and senior triathletes (height $1.74 \pm 0.04$ $\mathrm{m}$, weight $70.82 \pm 6.76 \mathrm{~kg}$, age $23.42 \pm 3.25$ years, $\mathrm{VO}_{2} \max 63.54 \pm 5.23 \mathrm{ml} \cdot$ $\mathrm{kg}-1 \cdot \min -1)$ participated in this study. They carried out three complete triathlons at different swimming intensities $(70 \%, 80 \%$ and $90 \%$ of a previous $750 \mathrm{~m}$ test). Heart rate and lactate were measured at the end of each sector and after completing the whole triathlon. Results: The $90 \%$ swimming intensity obtained the best final performance. Lactate and heart rate in the swimming sector for this condition increased significantly, without differences in the following sectors. Conclusions: Based on the sample studied, the final performance in a sprint triathlon seems to be conditioned by the swim intensity, being $90 \%$ the best intensity observed in moderately trained triathletes.
\end{abstract}

KEYWORDS: Endurance, performance, training.

\title{
INTRODUCCIÓN
}

El triatlón es un deporte multidisciplinar que involucra tres disciplinas sucesivas: natación, ciclismo y carrera a pie. Al mismo tiempo, el triatlón dispone de diferentes modalidades de competición, como son sprint, Olímpico y larga distancia, las cuales se caracterizan por diferentes distancias de competición. En relación a la investigación de este deporte, la inmensa mayoría de estudios de rendimiento en triatletas se han centrado en los efectos del uso de neopreno $(1,2,3)$, el uso del drafting en natación $(4,5,6,7,8,9)$, drafting en ciclismo $(16,17,18)$ y en el efecto del sector de ciclismo en la carrera a pie posterior $(10,11,12,13)$.

En triatlón, la disciplina de natación es importante porque el resultado final estará influenciado por el rendimiento de los triatletas en este sector. Landers et al. (14) encontraron que los ganadores de diferentes triatlones terminaron el sector de natación en el primer grupo en el $90 \%$ y $70 \%$ de las competiciones en hombres y mujeres, respectivamente. Cejuela et al. (15) mostraron que la disciplina de natación corresponde con el $\sim 16 \%$ del tiempo total del triatlón. También, el orden final durante la disciplina de natación (14) correlaciona con la posición final del triatlón ( $r=0,49$ y 0,39 , mujeres y hombres, respectivamente). Por lo tanto, podemos asumir que el sector de natación es muy importante para el resultado final del triatlón, y, en definitiva, analizar el ritmo óptimo de nado de este sector pueda influir en el resultado final.

Sin embargo, Vleck et al. (16) encontraron que rendimientos bajos en esta parte, provoca realizar un gran esfuerzo en el sector de ciclismo y, en definitiva, influye en la carrera a pie. Además, Peeling y Landers (17) enfatizan que la energía usada y la posición final durante el sector de natación es importante en 
determinar el éxito final de todo el triatlón. En este sentido, la intensidad a la que se realiza el nado, ha sido poco estudiada en la literatura científica. Por ejemplo, Peeling et al. (18) llevaron a cabo un estudio en el que los triatletas realizaron tres triatlones sprint bajo condiciones de laboratorio a intensidades del $80 \%, 90 \%$ y del $100 \%$ de un test de nado de la misma distancia sprint $(750 \mathrm{~m})$. Estos autores encontraron que la intensidad de nado al $80 \%$ redujo la frecuencia de ciclo al nadar, el lactato sanguíneo y el tiempo total del triatlón fue inferior al resto de intensidades.

Considerando por tanto la importancia de optimizar la intensidad a la que se realiza el sector de natación de cara al rendimiento final de un triatlón, el objetivo de este estudio es analizar la influencia de diferentes intensidades de nado $(70 \%$, $80 \%$ y $90 \%$ del test de nadoSTT) en el rendimiento posterior en el sector de ciclismo, carrera y el total del triatlón.

\section{MATERIAL Y MÉTODO}

\section{Sujetos}

Siete triatletas masculinos de categorías sub23 y Absolutos, moderadamente entrenados (altura de 1,74 $\pm 0,04 \mathrm{~m}$, peso de 70,82 $\pm 6,76 \mathrm{~kg}$, edad de 23,42 \pm 3,25 años, y $\mathrm{VO}_{2}$ max de $63,54 \pm 5,23 \mathrm{ml} \cdot \mathrm{kg}^{-1} \cdot \mathrm{min}^{-1}$ ) participaron en este estudio. Previo al estudio, los sujetos fueron informados de los test a realizar, de los posibles riesgos y tuvieron que firmar un consentimiento informado. Los datos fueron obtenidos y tratados en la Universidad de Castilla-La Mancha (España), entre los meses febrero-marzo del 2018, de forma anónima, para proteger la identidad de cada uno de los sujetos evaluados. Este estudio fue realizado de acuerdo a los principios de la Declaración de Helsinki.

\section{Procedimiento}

La investigación fue llevada a cabo durante cinco sesiones que implicaron un test incremental de $\mathrm{VO}_{2}$ max, un test de $750 \mathrm{~m}$ (STT) y tres triatlones distancia sprint (TRI) separados por al menos $72 \mathrm{~h}$ de descanso, y fueron llevados a cabo todos a la misma hora del día. Se les informó a los sujetos que no debían realizar ejercicio intenso $24 \mathrm{~h}$ antes de cada test. Todos los test de natación se llevaron a cabo en una piscina cubierta de seis calles, $25 \mathrm{~m}$ de longitud y bajo las mismas condiciones de temperatura (27ㅇ). El test incremental de $\mathrm{VO}_{2}$ max y el sector de ciclismo de cada triatlón, se realizaron en el Laboratorio bajo las mismas condiciones (550m altitud, $20-25$ - temperatura y $35-40 \%$ de humedad relativa).

En la primera sesión, un test incremental en cicloergómetro (Lode Excalibur Sport, Groningen, Holanda) para determinar $\mathrm{VO}_{2}$ max fue realizado. El test comenzó con 5 minutos de calentamiento a $75 \mathrm{~W}$ y, después la carga fue aumentada con incrementos de 50W cada minuto hasta la extenuación, siguiendo el protocolo de Craig et al. (19).

Tras 48h de recuperación, en la segunda sesión, un test de 750m de nado (STT) fue realizado en la piscina cubierta tras $200 \mathrm{~m}$ de calentamiento. Después, se les 
requirió a los sujetos a nadar $750 \mathrm{~m}$ tan rápido como fuera posible. El tiempo total, frecuencia cardíaca pico (Polar FT1, Polar Electro, Kempele, Finlandia), y lactato sanguíneo (Scout lactate analyzer, SensLab GmbH, Leipzig, Alemania) fueron medidos.

En las siguientes sesiones, tres TRI fueron realizados a diferentes intensidades de nado. Un calentamiento estándar de $200 \mathrm{~m}$ fue completado antes del inicio de cada sector de nado de cada triatlón (750m a intensidades del $70 \%, 80 \%$ y $90 \%$ de la velocidad del test STT), en un orden aleatorio. Con el fin de controlar bien el ritmo de nado, un metrónomo acuático (Finis Tempo Trainer) fue colocado en el interior del gorro de natación cerca del oído del nadador. Después del sector de natación, los sujetos se movieron al laboratorio para realizar el sector de ciclismo (situado a $50 \mathrm{~m}$ de la piscina). Los sujetos pedalearon en el cicloergómetro (SNT Medical Cardgirus, Bikemarc Sport Technology, Barcelona, España) una distancia de $20 \mathrm{~km}$ en el mejor tiempo posible. La cadencia y potencia media fue medida. Al finalizar el sector de ciclismo, los sujetos corrieron en el exterior del Laboratorio una distancia de $5 \mathrm{~km}$ en el menor tiempo posible. La frecuencia cardíaca pico, lactato sanguíneo y tiempo fue medido tras cada sector, y al finalizar el triatlón completo.

\section{Análisis estadístico}

Los resultados están expresados como media y desviación estándar. El test Shapiro-Wilk fue usado para comprobar la homogeneidad de cada variable $(p>0,05)$. Un análisis de medidas repetidas (ANOVA) fue inicialmente realizado para identificar las diferencias en las variables de estudio. El tamaño del efecto (ES) fue calculado para evaluar la magnitud de los cambios, el cual fue interpretado como pequeño $(<0,3)$, moderado $(\geq 0,3$ y $<0,5)$ y grande $(\geq 0,5)$. El análisis fue realizado usando SPSS (version 21.0, IBM Corp, New York, NY, USA) para Windows. La significancia estadística fue establecida en $p<0,05$.

\section{RESULTADOS}

Los resultados están expresados como media \pm SD y se muestran en la Tabla 1. A su vez, se muestra el porcentaje de contribución de cada uno de los sectores respecto al tiempo total en la Figura 1. 
Tabla 1. Resultados de las variables analizadas en función de la intensidad de nado

\begin{tabular}{|c|c|c|c|c|c|}
\hline \multirow[b]{2}{*}{ Variables } & \multicolumn{3}{|c|}{ \% Intensidad del sector de natación } & \multirow[b]{2}{*}{ Sig. } & \multirow[b]{2}{*}{ ES } \\
\hline & $70 \%$ (media \pm SD) & $80 \%$ (media \pm SD) & $90 \%$ (media \pm SD) & & \\
\hline Tiempo total triatlón (segundos) & $4364,14 \pm 253,52$ & $4290,57 \pm 241,92$ & $4179,85 \pm 226,17$ & $0,045^{*}$ & 0,403 \\
\hline Tiempo natación (segundos) & $953,14 \pm 170,46$ & $849,28 \pm 124,29$ & $744,28 \pm 117,57$ & $0,0001^{* *}$ & 0,896 \\
\hline Tiempo ciclismo (segundos) & $2227,71 \pm 132,69$ & $2246,71 \pm 136,70$ & $2192,57 \pm 149,55$ & 0,56 & 0,092 \\
\hline Tiempo carrera a pie (segundos) & $1185,14 \pm 66,56$ & $1194,85 \pm 87,84$ & $1225,42 \pm 65,39$ & 0,115 & 0,303 \\
\hline Lactato natación $\left(\mathrm{mmol} \mathrm{I}^{-1}\right)$ & $2,21 \pm 0,59$ & $3,22 \pm 0,72$ & $5,44 \pm 1,97$ & $0,0001^{\star *}$ & 0,742 \\
\hline Lactato ciclismo $\left(\mathrm{mmol} \mathrm{I}^{-1}\right)$ & $5,88 \pm 3,95$ & $5,12 \pm 2,57$ & $4,85 \pm 3,07$ & 0,725 & 0,052 \\
\hline Lactato carrera a pie $\left(\mathrm{mmol} \mathrm{I}^{-1}\right)$ & $8,04 \pm 4,21$ & $7,38 \pm 2,57$ & $6,12 \pm 2,40$ & 0,07 & 0,357 \\
\hline FC natación (pulsaciones/minuto) & $144,85 \pm 13,32$ & $120,14 \pm 16,64$ & $132,28 \pm 25,95$ & $0,046^{*}$ & 0,401 \\
\hline FC ciclismo (pulsaciones/minuto) & $155,71 \pm 23,31$ & $161,42 \pm 21,61$ & $152,57 \pm 19,87$ & 0,222 & 0,222 \\
\hline FC carrera a pie (pulsaciones/minuto) & $165,28 \pm 13,05$ & $154,71 \pm 17,65$ & $160,14 \pm 17,47$ & 0,287 & 0,18 \\
\hline Potencia (watios) & $171,71 \pm 24,87$ & $165,28 \pm 23,69$ & $171,71 \pm 28,65$ & 0,784 & 0,04 \\
\hline Cadencia (revoluciones por minuto) & $98,71 \pm 8,77$ & $99,57 \pm 2,93$ & $99,43 \pm 3,60$ & 0,947 & 0,009 \\
\hline
\end{tabular}

* Diferencias significativas para $p<0,05$; ${ }^{* *}$ Diferencias significativas para $p<0,01$; ES, tamaño del efecto (d Cohen)

\section{Tiempo total de los triatlones}

La media de tiempo de los triatlones al $70 \%, 80 \%$ y $90 \%$ del STT fue de 4364,14 $\pm 253,52,4290,57 \pm 241,92$ y $4179,85 \pm 226,17$ segundos. El tiempo empleado en la realización del triatlón en la condición de nado del 90\% STT fue menor en comparación al $70 \%$ y $80 \%$ STT ( $<<0,05)$.

\section{Sector de natación}

El tiempo total de este sector para los tres triatlones fue de 953,14 $\pm 170,46$, $849,28 \pm 124,29$ y $744,28 \pm 117,57$, en las condiciones del $70 \%, 80 \%$ y $90 \%$ STT, respectivamente. El lactato sanguíneo tras la realización de este sector fue de $2,21 \pm 0,59,3,22 \pm 0,72$ y $5,44 \pm 1,97 \mathrm{mmol} \mathrm{l}^{-1}$, en las condiciones del $70 \%, 80 \%$ y $90 \%$ STT, respectivamente, siendo superior en $90 \%$ STT $(p<0,001)$ en comparación con $70 \%$ y $80 \%$ STT. En lo que respecta a la frecuencia cardíaca, ésta fue significativamente superior $(p<0,05)$ en la condición $90 \%$ STT $(144,85 \pm$ $13,32,120,14 \pm 16,64$ y $132,28 \pm 25,95$ pulsaciones por minuto) comparado con $70 \%$ y $80 \%$ STT, respectivamente.

\section{Sector de ciclismo}

El tiempo total de este sector para los tres triatlones fue de $2227,71 \pm 132,69$, $2246,71 \pm 136,70$ y $2192,57 \pm 149,55$ segundos, en las condiciones del $70 \%$, $80 \%$ y $90 \%$ STT, respectivamente, sin mostrar diferencias entre grupos. El lactato sanguíneo se mantuvo sin diferencias significativas entre condiciones $\left(5,88 \pm 3,95,5,12 \pm 2,57\right.$ y $4,85 \pm 3,07, \mathrm{mmol} \mathrm{I}^{-1}$ respectivamente). En relación a la frecuencia cardíaca, ésta se mantuvo sin cambios en las tres condiciones $(155,71 \pm 23,31,161,42 \pm 21,61$ y $152,57 \pm 19,87$ pulsaciones por minuto, al $70 \%, 80 \%$ y $90 \%$ STT, respectivamente). En lo que respecta a los parámetros 
de potencia y cadencia en este sector, se mantuvieron sin diferencias entre condiciones.

\section{Sector de carrera a pie}

El tiempo total de este sector fue de $1185,14 \pm 66,56,1194,85 \pm 87,84$ y 1225,42 $\pm 65,39$ segundos, en las condiciones del $70 \%, 80 \%$ y $90 \%$ STT, respectivamente, sin mostrar diferencias entre grupos. En lo que respecta al lactato sanguíneo $\left(8,04 \pm 4,21 ; 7,38 \pm 2,57\right.$ y $6,12 \pm 2,40 \mathrm{mmol} \mathrm{I}^{-1}$, en las condiciones del $70 \%, 80 \%$ y $90 \%$ STT, respectivamente) no se encontraron diferencias significativas $(\mathrm{p}=0,07)$, pero sí un tamaño del efecto pequeño $(E S=0,35)$. Finalmente, la frecuencia cardíaca se mantuvo sin cambios en las tres condiciones experimentales $(165,28 \pm 13,05,154,71 \pm 17,65$ y $160,14 \pm$ 17,47 pulsaciones por minuto, en las condiciones del $70 \%, 80 \%$ y $90 \%$ STT, respectivamente).

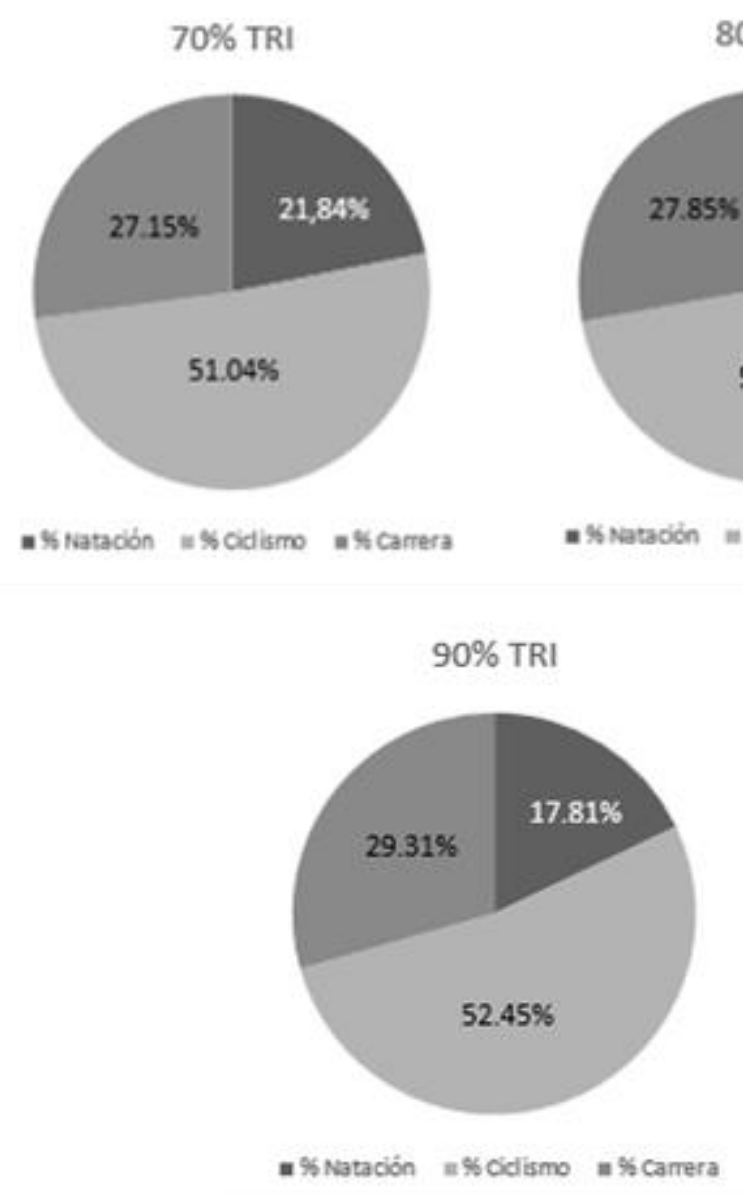

Figura 1. Contribución de cada sector relativo al tiempo total del triatión en función de cada intensidad de nado.

\section{DISCUSIÓN}

El objetivo de este estudio fue analizar la influencia de diferentes intensidades de nado en el rendimiento posterior de ciclismo, carrera a pie y el tiempo total de 
un triatlón sprint, así como su efecto en la frecuencia cardíaca y lactato sanguíneo. El resultado más destacado fue que la intensidad de nado del $90 \%$ STT fue la que mejor resultado obtuvo en el tiempo total para completar el triatlón. Nuestro resultado se diferencia de los resultados encontrados por Peeling et al. (18), cuyos autores encontraron que la intensidad del $80 \%$ STT fue la que mejor rendimiento final obtuvo. Estas discrepancias pueden deberse al nivel de la muestra (altamente entrenados vs moderadamente entrenados como en nuestro estudio). A diferencia de otros estudios, este estudio analiza intensidades más bajas que no han sido mostradas anteriormente por Peeling et al. (19) y Vleck et al. (16), como es la intensidad del $70 \%$ de un test de la distancia propia del sector de natación en un triatlón sprint $(750 \mathrm{~m})$.

Por otro lado, de acuerdo a las conclusiones de Peeling et al. (19), una intensidad baja de nado influiría en un mejor rendimiento posterior en el sector de ciclismo y carrera, aunque en nuestro estudio no ocurriese lo mismo. No encontramos diferencias significativas entre condiciones en los tiempos totales de los sectores de ciclismo y carrera. A su vez, las conclusiones de Peeling et al. $(17,18)$ difieren de las encontradas por Vleck et al. (16). De acuerdo a este autor, parece que un rendimiento inferior en natación, puede resultar en un gran esfuerzo físico al inicio del sector de ciclismo, y podría influir en el posterior sector de carrera. A pesar de esto, en nuestro estudio no encontramos diferencias significativas en el tiempo y en el lactato sanguíneo en carrera, aunque el tamaño del efecto moderado ( $E S=0,303$ y 0,357 , respectivamente) en estas variables indica una tendencia a que, a mayor intensidad de nado, el triatleta no es capaz de desplegar su mejor rendimiento en ese sector. Tal vez con un mayor número de sujetos, esta tendencia se hubiera mostrado significativa. Nuestros resultados mostraron una relación directa entre el tiempo en el sector de carrera y el lactato sanguíneo resultante, puesto que los sujetos cuando nadaron a menor intensidad (70\% TRI), fueron capaces de correr más rápido el sector a pie, y, por lo tanto, el lactato sanguíneo era superior, aunque no significativo $(E S=0,357)$, mostrando dicha tendencia.

En relación a los resultados durante el sector de natación, se encontraron diferencias significativas en el lactato sanguíneo y frecuencia cardíaca. La condición $90 \% \mathrm{TRI}$ alcanzó un mayor valor en lactato sanguíneo y frecuencia cardíaca. Estudios previos han mostrado una alta correlación entre lactato sanguíneo y frecuencia cardíaca, mostrando que, a una mayor intensidad, el lactato y la frecuencia cardíaca incrementa en la misma proporción $(20,21)$.

Nuestros resultados muestran que el tiempo final en el triatlón es mejorado cuando el sector de natación se nadó más rápido (90\% TRI), a diferencia de lo encontrado por Kreider et al. (22) y Peeling et al. (18), cuyos resultados mostraron que una baja intensidad relativa en el sector de natación podría implicar un incremento en el rendimiento de los sectores de ciclismo y carrera. Otros autores han mostrado como no sólo la intensidad de nado influye en el rendimiento final del triatlón, sino también la estrategia de nado utilizada, siendo la estrategia positiva la que mejor rendimiento final (23). Sin embargo, en nuestro estudio no encontramos diferencias significativas entre condiciones de intensidades de nado en los sectores de ciclismo y carrera, aunque el tiempo medio de la condición $90 \%$ TRI en el sector de carrera fue ligeramente superior 
en comparación al $70 \%$ y $80 \%$ TRI, pero sin ser significativo ( $p=0,115)$. El tamaño del efecto moderado $(E S=0,303)$ puede mostrarnos una tendencia a que una intensidad superior en el sector de nado, podría reducir el rendimiento en el sector de carrera. Teniendo esto en cuenta, el tiempo ganado en el sector de nado cuando se realiza a una intensidad superior ( $90 \%$ STT), es suficiente para obtener un tiempo total del triatlón mejor, pese a la pérdida de tiempo en el sector de carrera en triatletas moderadamente entrenados. De esta manera, en triatletas entrenados, la intensidad óptima de nado estaría entre el $80-90 \%$, de acuerdo a nuestros resultados y los previamente mostrados por Peeling et al. (18).

\section{CONCLUSIONES}

El presente estudio concluye que la intensidad de nado es determinante para el rendimiento final de un triatlón de distancia sprint en la muestra estudiada. Sin embargo, se recomienda estudios adicionales con una mayor muestra y en triatletas de un nivel de rendimiento superior.

\section{REFERENCIAS BIBLIOGRÁFICAS}

1. Chatard JC, Senegas X, Selles M, Dreanot P, Geyssant A. Wet suit effect: a comparison between competitive swimmers and triathletes. Med Sci Sport Exer. 1995;27(4):580-586. https://doi.org/10.1249/00005768-199504000$\underline{00017}$

2. Cordain L, Kopriva R. Wetsuits, body density and swimming performance. Brit J Sport Med. 1991;25(1):31-33. https://doi.org/10.1136/bjsm.25.1.31

3. De Lucas RD, Balikian P, Neiva CM, Greco CC, Denadai BS. The effects of wet suits on physiological and biomechanical indices during swimming. J Sci Med Sport. 2000;3(1):1-8. https://doi.org/10.1016/S1440-2440(00)80042-0

4. Bassett Jr DR, Flohr J, Duey WJ, Howley ET, Pein RL. Metabolic responses to drafting during front crawl swimming. Med Sci Sport Exer. 1991;23(6):744747. https://doi.org/10.1249/00005768-199106000-00015

5. Bassett DR, Howley ET. Limiting factors for maximum oxygen uptake and determinants of endurance performance. Med Sci Sport Exer. 2000;32(1):7084. https://doi.org/10.1097/00005768-200001000-00012

6. Chatard JC, Chollet D, Millet G. Performance and drag during drafting swimming in highly trained triathletes. Med Sci Sport Exer. 1998;30(8):12761280. https://doi.org/10.1097/00005768-199808000-00015

7. Chollet D, Hue O, Auclair F, Millet G, Chatard JC. The effects of drafting on stroking variations during swimming in elite male triathletes. Eur $\mathrm{J}$ Appl Physiol. 2000;82(5-6):413-417. https://doi.org/10.1007/s004210000233

8. Delextrat A, Tricot V, Bernard T, Vercruyssen F, Hausswirth C, Brisswalter J. Modification of cycling biomechanics during a swim-to-cycle trial. J Appl Biomech. 2005;21(3):297-308. https://doi.org/10.1123/jab.21.3.297

9. Millet GP, Vleck VE. Physiological and biomechanical adaptations to the cycle to run transition in Olympic triathlon: review and practical recommendations for training. Brit J Sport Med. 2000;34(5):384-390. https://doi.org/10.1136/bjsm.34.5.384

10. Bonacci J, Blanch P, Chapman AR, Vicenzino B. Altered movement patterns but not muscle recruitment in moderately trained triathletes during running 
after cycling. J Sports Sci. 2010;28(13):1477-1487. https://doi.org/10.1080/02640414.2010.514279

11. Bonacci J, Saunders PU, Alexander $M$, Blanch $P$, Vicenzino $B$. Neuromuscular control and running economy is preserved in elite international triathletes after cycling. Sport Biomech. 2011;10(01):59-71. https://doi.org/10.1080/14763141.2010.547593

12. Etxebarria N, Anson JM, Pyne DB, Ferguson RA. Cycling attributes that enhance running performance after the cycle section in triathlon. Int J Sports Physiol Perform. 2013;8(5):502-509. https://doi.org/10.1123/ijspp.8.5.502

13. Vercruyssen F, Brisswalter J, Hausswirth C, Bernard T, Bernard O, Vallier JM. Influence of cycling cadence on subsequent running performance in triathletes. Med Sci Sport Exer. 2002;34(3):530-536. https://doi.org/10.1097/00005768-200203000-00022

14. Landers GJ, Blanksby BA, Ackland TR, Monson R. Swim Positioning and its Influence on Triathlon Outcome. Int J Exerc Sci. 2008;1(3):96-105.

15. Cejuela R, Cala A, Pérez-Turpin JA, Villa JG, Cortell JM, Chinchilla JJ. Temporal activity in particular segments and transitions in the olympic triathlon. J Hum Kinet. 2013;36(1):87-95. https://doi.org/10.2478/hukin-20130009

16. Vleck VE, Bürgi $A$, Bentley DJ. The consequences of swim, cycle, and run performance on overall result in elite Olympic distance triathlon. Int J Sport Med. 2006;27(01):43-48. https://doi.org/10.1055/s-2005-837502

17. Peeling $P$, Landers $G$. Swimming intensity during triathlon: a review of current research and strategies to enhance race performance. J Sport Sci. 2009;27(10):1079-1085. https://doi.org/10.1080/02640410903081878

18. Peeling PD, Bishop DJ, Landers GJ. Effect of swimming intensity on subsequent cycling and overall triathlon performance. Brit $\mathrm{J}$ Sport Med. 2005;39(12):960-964. https://doi.org/10.1136/bjsm.2005.020370

19. Craig N, Walsh C, Martin DT, Woolford S, Bourdon P, Stanef T, Savage B. Protocols for the physiological assessment of high-performance track, road and mountain cyclists. Physiological tests for elite athletes/Australian Sports Commission. Champaign (IL): Human Kinetics, 2000:258-77.

20. Belcher CP, Pemberton CL. The Use of the Blood Lactate Curve to Develop Training Intensity Guidelines for the Sports of Track and Field and CrossCountry. Int $J$ of Exerc Sci. 2012;5(2):148-159. https://doi.org/10.1123/ice.6.2.148

21. Lopes RF, Osiecki R, Rama LMPL. Heart rate and blood lactate concentration response after each segment of the Olympic Triathlon event. Rev Bra Med Esporte. 2012;18(3):158-160. https://doi.org/10.1590/S151786922012000300003

22. Kreider RB, Boone T, Thompson WR, Burkes S, Cortes CW. Cardiovascular and thermal responses of triathlon performance. Med Sci Sport Exer. 1988;20(4):385-390. https://doi.org/10.1249/00005768-198808000-00010

23. Wu SS, Peiffer JJ, Peeling P, Brisswalter J, Lau WY, Nosaka K, Abbiss CR. Improvement of sprint triathlon performance in trained athletes with positive swim pacing. Int $J$ Sports Physiol Perform. 2016;11(8):1024-1028. https://doi.org/10.1123/ijspp.2015-0580 
Rev.int.med.cienc.act.fís.deporte - vol. 20 - número 80 - ISSN: 1577-0354

Número de citas totales/Total references: 23 (100\%)

Número de citas propias de la revista/Journal's own references: $0(0 \%)$

Rev.int.med.cienc.act.fís.deporte - vol. X - número X - ISSN: 1577-0354 